\title{
MSNA during prolonged post-faint hypotension
}

\author{
J. Rozenberg $\cdot$ W. Wieling $\cdot$ I. K. Schon \\ B. Westerhof · C. Frampton · D. Jardine
}

Received: 28 November 2011 / Accepted: 14 February 2012/Published online: 14 March 2012

(C) The Author(s) 2012. This article is published with open access at Springerlink.com

\begin{abstract}
Background Following tilt-induced syncope, blood pressure usually recovers rapidly after tilt back to the horizontal position. However, in some patients, hemodynamic recovery is delayed, a condition recently termed "prolonged postfaint hypotension" (PPFH). The mechanism is thought to be mediated by increased vagal outflow rather than exaggerated peripheral vasodilatation and sympathetic withdrawal. To date, no muscle sympathetic nerve activity (MSNA) recordings have been reported in this condition, so we aimed to confirm that neither vasodilatation nor MSNA withdrawal was responsible.

Objectives To retrospectively select patients with satisfactory recordings of continuous BP and MSNA during tiltinduced syncope. To compare hemodynamic and MSNA profiles in patients with PPFH to patients with normal recovery (NR) after tilt-back.

Methods All patients were studied in Christchurch, New Zealand, between 1998 and 2008 using continuous arterial
\end{abstract}

J. Rozenberg · W. Wieling

Department of Internal Medicine, Academic Medical Center,

University of Amsterdam, Amsterdam, The Netherlands

J. Rozenberg $(\square)$

De Clercqstraat 43-II, 1053 AC Amsterdam, The Netherlands

e-mail: Josien.Rozenberg@student.uva.nl

I. K. Schon - B. Westerhof

BMEYE, Academic Medical Center, Amsterdam,

The Netherlands

C. Frampton · D. Jardine

Department of Medicine, Christchurch Hospital,

Christchurch, New Zealand
BP monitoring, and microneurographic recordings of MSNA from the right leg. Only patients with satisfactory BP and MSNA data throughout baseline, head-up tilt and presyncope were selected. Stroke volume (SV), cardiac output (CO), and total peripheral resistance (TPR) were derived using Modelflow. After baseline measurements, patients were tilted to the head-up $60^{\circ}$ position and given GTN spray if asymptomatic after $20 \mathrm{~min}$. Following the onset of presyncope, patients were tilted slowly back to the horizontal. PPFH was defined as systolic $\mathrm{BP}<85 \mathrm{mmHg}$ for at least 2 min after tilt-back. Measurements were averaged at baseline, early tilt, presyncope, early and late recovery. Within-group comparisons were made between baseline and all other time points. Between-group comparisons were made over all time points.

Results Patients with PPFH (7 males, age $46 \pm 5$ years, $n=8$ ) and with NR (8 males, age $47 \pm 6$ years, $n=8$ ) were selected. Presyncope was provoked by GTN in $4 / 8$ patients in each group. In both groups, MAP remained below baseline during early and late recovery: PPFH $84 \pm 5$ versus $51 \pm 5$ and $64 \pm 5 \mathrm{mmHg}(p=0.001$, $p=0.001) ; \mathrm{NR} 104 \pm 5$ versus $83 \pm 5$ and $93 \pm 5$ $\mathrm{mmHg}(p=0.001, p=0.03)$. However, MAP and HR were lower in the PPFH group $(p=0.004, p=0.023)$. During early recovery, $\mathrm{CO}$ remained below baseline only in the PPFH group $(p=0.001)$, whereas TPR remained constant in both groups. In both groups, all MSNA indices tended to remain above baseline levels during early and late recovery. PPFH $25 \pm 2$ increased to $31 \pm 6$ and $29 \pm 4$ bursts $/ \min (p=0.09,0.02)$; NR $23 \pm 3$ increased to $33 \pm 3$ and $34 \pm 3$ bursts $/ \min (p=0.06,0.01)$.

Conclusions PPFH does not appear to be mediated by exaggerated vasodilatation or sympathetic withdrawal. Delayed recovery of cardiac output by increased vagal outflow is a more likely mechanism. 
Keywords Prolonged post faint hypotension (PPFH) . Muscle sympathetic nerve activity (MSNA) - Total peripheral resistance (TPR) · Cardiac output (CO) · Sympathetic withdrawal · Vagal outflow

\section{Introduction}

Until recently, the mechanism of vasovagal syncope was thought to be dual, vasodilatation mediated by sympathetic withdrawal and bradycardia mediated by vagal activation [1]. The concept of sudden reduction of sympathetic activity during faint causing relaxation of the blood vessels was based on plethysmographic demonstration of forearm vasodilatation [2-4], and microneurographic recordings showing withdrawal of muscle sympathetic nerve activity (MSNA) in the leg [5-9]. MSNA is considered to be a major determinant of rapid changes in total peripheral resistance (TPR) [10, 11]. Despite these important findings, other studies found that peripheral vasodilatation was not generalized and probably a terminal event in the time-course of the vasovagal reaction. Invasive studies [12-15] demonstrated that hypotension during presyncope was initiated by a fall in cardiac output which began up to $5 \mathrm{~min}$ before syncope. Continuous finger-pulse BP monitoring with Modelflow, confirmed that a gradual fall in cardiac output, secondary to stroke volume decay mediated progressive hypotension during presyncope [16]. This was consistent with the observation that presyncope could be rapidly reversed by maneuvers, which increased venous return and stroke volume [17, 18]. Therefore, decreasing cardiac output is clearly important in the sequence of hemodynamic changes before vasovagal syncope. Recently, Wieling et al. [19] described a new group of patients with a history of severe fainting. Continuous BP monitoring during tilt-induced syncope demonstrated prolonged post faint hypotension (PPFH) and the mechanism for this appeared to be mainly delayed recovery of cardiac output and reduced left ventricular contractility. TPR was maintained throughout the faint and so even in PPFH patients with severe fainting, no peripheral sympathetic withdrawal was found. So far, no MSNA measurements have been performed in PPFH patients. We hypothesized that because TPR was maintained during PPFH, MSNA would also remain increased from baseline values. We also hypothesized that PPFH patients might have lower resting TPR and MSNA levels compared to NR patients, predisposing them to more severe episodes of VVS.

\section{Methods}

\section{Study population and protocol}

We reviewed hemodynamic data from 340 recordings of tilt tests undertaken in the Christchurch Hospital Syncope
Clinic from 1995 to 2005. All patients had a history of vasovagal symptoms over the 6 months before tilt. Of these, 203 tilt tests were positive. A positive tilt test was defined as a progressive fall in systolic blood pressure (SBP) below $100 \mathrm{mmHg}$ associated with symptoms of impending syncope. PPFH, defined as $\mathrm{SBP}<85 \mathrm{mmHg}$ for at least 2 min after tilt back was found in 25 patients $(7.4 \%$ of tilt tests). From this group, on the basis of satisfactory MSNA recordings during tilt and presyncope (see below), we selected eight PPFH patients, ( 7 males, age $46 \pm 5$ years). Nearly all had mild bradycardia during late recovery (in 7 out of 8 , HR fell below $60 \mathrm{bpm}$ ). However, only two of our PPFH patients had severe bradycardia (HR less than $50 \mathrm{bpm}$ ) as in the group described by Wieling et al. [19]. A further eight patients with satisfactory MSNA recordings and normal recovery (NR) of blood pressure after tilt-induced syncope were selected as controls (8 males, age $47 \pm 6$ years). Patients were included in the NR group provided SBP increased to $>85 \mathrm{mmHg}$ during the first 2 min of recovery.

Passive head-up tilt (HUT) to $60^{\circ}$ was performed on a hydraulic tilt-table between 9:00 AM and 1:00 PM in a temperature-controlled room $\left(23^{\circ} \mathrm{C}\right)$ according to the Italian protocol [20]. If presyncope did not occur within $20 \mathrm{~min}$, nitroglycerine $(0.4 \mathrm{mg})$ was administered sublingually. When a progressive fall in blood pressure occurred, associated with prodromal symptoms, the patient was tilted slowly back to the horizontal over $20 \mathrm{~s}$.

This study was approved by the Canterbury Hospital Ethics Committee.

Data acquisition and analysis

Microneurography needles were positioned for recording MSNA from the right peroneal nerve [21]. The nerve was located behind the head of the fibula, and, with the use of transcutaneous stimulation, an insulated tungsten electrode with a 1- to 5- $\mu \mathrm{m}$ tip was inserted. The nerve signal was amplified $(1,00,000 \times)$ filtered $(700-2,000 \mathrm{~Hz})$, integrated (time constant $0.1 \mathrm{~ms}$ ), and displayed online with blood pressure and ECG. Bursts of sympathetic activity were indentified and counted each minute (bs/min) by the same operator (DLJ). The nerve signal was accepted if the following criteria were met: the signal-to-background ratio was greater than 3; the bursts were pulse-synchronous; burst amplitude was inversely proportional to diastolic BP; and skin activity was absent. It is difficult to maintain the recording field during recovery following the tilt-back procedure particularly when patients are severely hypotensive and symptomatic. Therefore, although we were able to maintain MSNA recordings throughout recovery in all of the selected NR patients, we only achieved this in $50 \%$ of the PPFH group. 
Non-invasive beat-to-beat BP was measured at the finger (Finometer Blood Pressure Monitor) with the hand held at heart level in a sling. Invasive BP recordings were undertaken from a 3 French brachial arterial line with a deltrans transducer fixed at mid-atrial level. The measured signal was analogue to digital converted at $200 \mathrm{~Hz}$, and stored on a hard disk for offline analysis. Systolic (SYS) and diastolic (DIAS) arterial pressures were derived from the arterial pressure waveform. Mean arterial pressure (MAP) was calculated from the integral of the arterial pressure wave over one beat divided by the corresponding beat interval. Heart rate (HR) was computed as the inverse of the inter-beat interval and expressed as beats per minute. Beat-to-beat changes in stroke volume (SV) were estimated by modeling flow from the arterial pressure waveform (Modelflow, TNO Biomedical Instrumentation), expressed in $\mathrm{ml}$ [22]. Cardiac output (CO), expressed in $1 / \mathrm{min}$, was the product of estimated SV and HR. Total Peripheral Resistance (TPR), expressed in $\mathrm{mmHg} \mathrm{s} / \mathrm{ml}$, as Medical units (MU) was derived from MAP divided by the computed CO. The Modelflow method estimates aortic flow from finger arterial pressure by simulating a non-linear model of the aortic input impedance [23]. This pulse wave analysis method corrects for individual differences in age, gender, height and weight, permitting group average data to be examined accurately in subjects without structural or functional heart disease [24]. Previous head-up tilt experiments have demonstrated good agreement between Modelflow and traditional estimates of CO [25, 26].

Sampling of time intervals

For each interval, hemodynamic variables were initially averaged over $30-\mathrm{s}$ periods and then averaged $(2 \times 30 \mathrm{~s}$ periods for each minute) for statistical testing. MSNA variables were initially counted and measured over 60-s periods. Values for all variables are expressed as mean $\pm \mathrm{SE}$.

The following time intervals were analyzed:

1. Baseline: the last $5 \mathrm{~min}$ in the supine position before head-up tilt

2. Early HUT: the steady-state adjustment to orthostatic stress during the 1-3 min of HUT (60-180 s).

3. Presyncope: the last minute before tilt-back to the horizontal position.

4. Recovery: 0-2 and 4-5 min following tilt-back to the horizontal position.

Statistical analysis

Statistical analysis was performed using unpaired $t$ tests and $\chi^{2}$ tests for demography and baseline variables.
Changes in variables with time were analyzed using oneway ANOVA for within-group and two-way ANOVA for between-group comparisons.

\section{Results (see Table 1 and Figs. 1, 2, 3)}

All patients had satisfactory MSNA data during tilt and presyncope, but 4/8 in the PPFH group had incomplete data during recovery. The groups were matched for age and gender. Four patients with PPFH (and 2 with NR) had a life-long history of syncope associated with blood phobia $(p=0.6)$. Five PPFH patients reported protracted symptoms after fainting (feeling unwell for more than $6 \mathrm{~h}$ ). Tilt time and requirement for GTN provocation were similar between groups.

Between group comparisons of haemodynamic parameters across all time points showed that MAP ( $p=0.004)$, and HR ( $p=0.023$ ) were lower in the in the PPFH group whereas SV was higher $(p=0.037)$. For all other variables, including CO $(p=0.89)$ and TPR $(p=0.128)$ and indices of MSNA (bursts/min $p=0.77$, bursts $/ 100 \mathrm{~b}$ $p=0.65$, and burst area/min $p=0.78$ ) there were no significant differences between groups.

\section{Baseline}

Between groups, the PPFH group had lower baseline BP and TPR than the NR group: MAP $84 \pm 4$ versus

Table 1 Patient characteristics and baseline hemodynamics

\begin{tabular}{llll}
\hline Variable & PPFH $(n=8)$ & NR $(n=8)$ & $p$ \\
\hline Age (years) & $46 \pm 5$ & $47 \pm 6$ & 1.0 \\
Gender (m) & 7 & 8 & 1.0 \\
Blood phobia & 4 & 2 & 0.6 \\
Syncopes per year & $2 \pm 0.7$ & $2.8 \pm 0.8$ & 0.5 \\
Tilt time (min) & $18.5 \pm 2$ & $20.3 \pm 3$ & 0.6 \\
GTN & 4 & 4 & 1.0 \\
MAP (mmHg) & $84 \pm 4$ & $104 \pm 5$ & 0.01 \\
HR (bpm) & $59 \pm 2$ & $73 \pm 6$ & 0.07 \\
SV (ml) & $102 \pm 8$ & $77 \pm 8$ & 0.05 \\
CO (1/min) & $5.9 \pm 0.5$ & $5.5 \pm 0.5$ & 0.59 \\
TPR (MU) & $0.9 \pm 0.1$ & $1.4 \pm 0.2$ & 0.05 \\
MSNA (bursts/min) & $25 \pm 2$ & $23 \pm 3$ & 0.8 \\
MSNA (bursts/100b) & $43 \pm 4$ & $35 \pm 7$ & 0.2 \\
\hline Mean \pm SE for demographics and & &
\end{tabular}

Mean \pm SE for demographics and baseline variables. All values quoted without units refer to absolute counts

$P P F H$ prolonged post-faint hypotension, NR normal recovery, GTN glyceryl trinitrate given at $20 \mathrm{~min}$ head-up tilt, tilt time time tilted up before presyncope, $M A P$ mean arterial pressure, $H R$ heart rate, $S V$ stroke volume, $C O$ cardiac output, $T P R$ total peripheral resistance, $M S N A$ muscle sympathetic nerve activity 


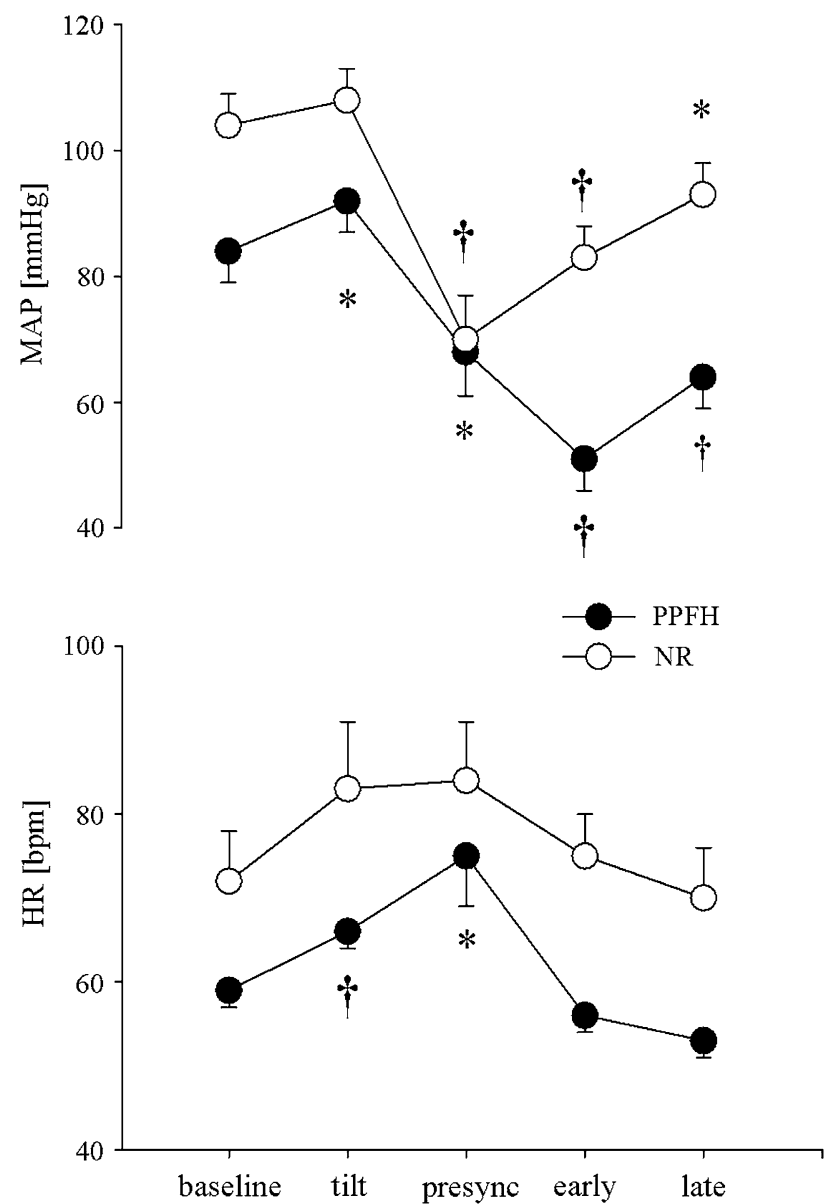

Fig. 1 Mean and standard error for mean arterial pressure and heart rate at baseline, early tilt, presyncope, early and late recovery in patients with prolonged post-faint hypotension (PPFH filled circles) and patients with normal recovery (NR open circles). Asterisks and dagger refer to $p<0.05$ and $p<0.01$ for within-group comparisons to baseline

$104 \pm 5 \mathrm{mmHg}(p=0.012)$; TPR $0.92 \pm 0.1$ versus $1.4 \pm$ $0.3 \mathrm{MU}(p=0.049)$, whereas SV was higher: $103 \pm 10$ versus $77 \pm 4 \mathrm{ml}(p=0.046)$. $\mathrm{HR}, \mathrm{CO}$ and all indices of MSNA were similar.

\section{Early HUT}

In the PPFH group MAP and HR increased to $92 \pm 5$ $\mathrm{mmHg}(p=0.043)$ and $66 \pm 2 \mathrm{bpm}(p=0.006)$ respectively, while SV and CO decreased to $75 \mathrm{ml}$ and $4.91 / \mathrm{min}$ $(p=0.004, p=0.004)$. TPR tended to increase from $0.92 \pm 0.1$ to $1.2 \pm 0.1 \mathrm{MU}(p=0.15)$. In the NR group, MAP remained constant at $108 \pm 5 \mathrm{mmHg}(p=0.2)$, while HR increased to $84 \pm 6 \mathrm{bpm}(0.02)$. SV and CO decreased to $54 \pm 6 \mathrm{ml}(p=0.004)$ and $4.3 \pm 0.4 \mathrm{l} / \mathrm{min}$ $(p=0.02)$ while TPR tended to increase $(p=0.1)$. MSNA indices increased in both groups: PPFH $25 \pm 2$ to $44 \pm 3$ bursts/min $(p=0.001), 43 \pm 4$ to $75 \pm 4$ bursts/100beats $(p=0.001)$ and 100 to $154 \pm 7 \%$ burst area $(p=0.001)$;
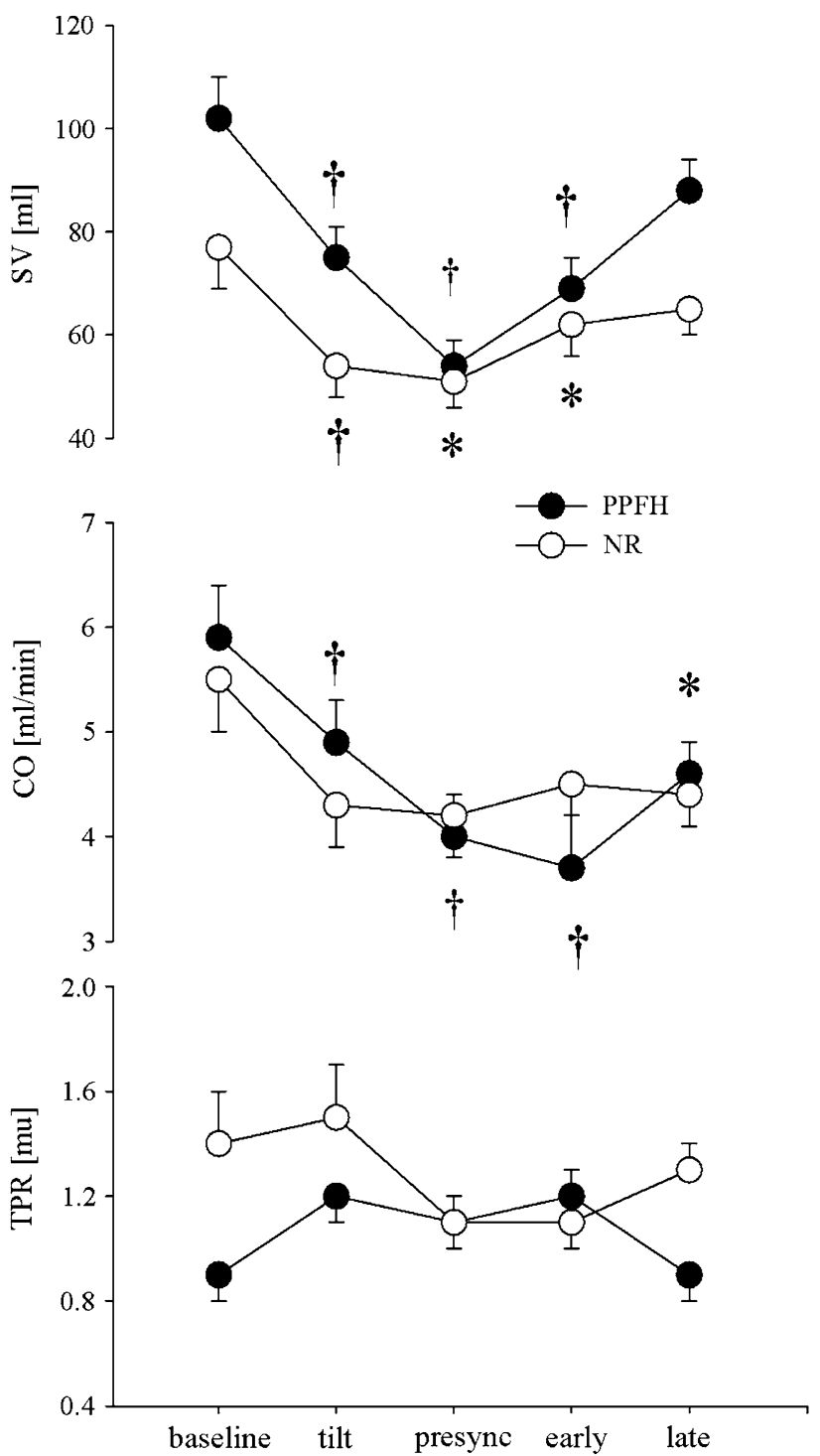

Fig. 2 Mean and standard error for stroke volume, cardiac output and total peripheral resistance at baseline, early tilt, presyncope, early and late recovery in patients with prolonged post-faint hypotension (PPFH filled circles) and patients with normal recovery (NR open circles). Asterisks and dagger refer to $p<0.05$ and $p<0.01$ for within-group comparisons to baseline

NR $23 \pm 3$ to $41 \pm 4$ bursts $/ \mathrm{min}(p=0.001), 35 \pm 7$ to $59 \pm 8$ bursts/100beats $(p=0.001)$ and 100 to $170 \pm$ $13 \%$ burst area $(p=0.001)$.

Presyncope (last minute before tilt back)

MAP fell below baseline levels in both groups: PPFH $68 \pm 7(p=0.045)$ and NR $70 \pm 7 \mathrm{mmHg}(p=0.002)$. In the PPFH group HR increased to $75 \pm 6 \mathrm{bpm}$ $(p=0.027), \mathrm{SV}$ fell to $54 \mathrm{ml}(p=0.003)$, and CO to $4.0 \mathrm{l} /$ $\min (p=0.002)$. In the NR group, SV fell further from baseline to $51 \mathrm{ml}(p=0.012)$. MSNA indices began to fall but remained above baseline levels in both groups: PPFH 

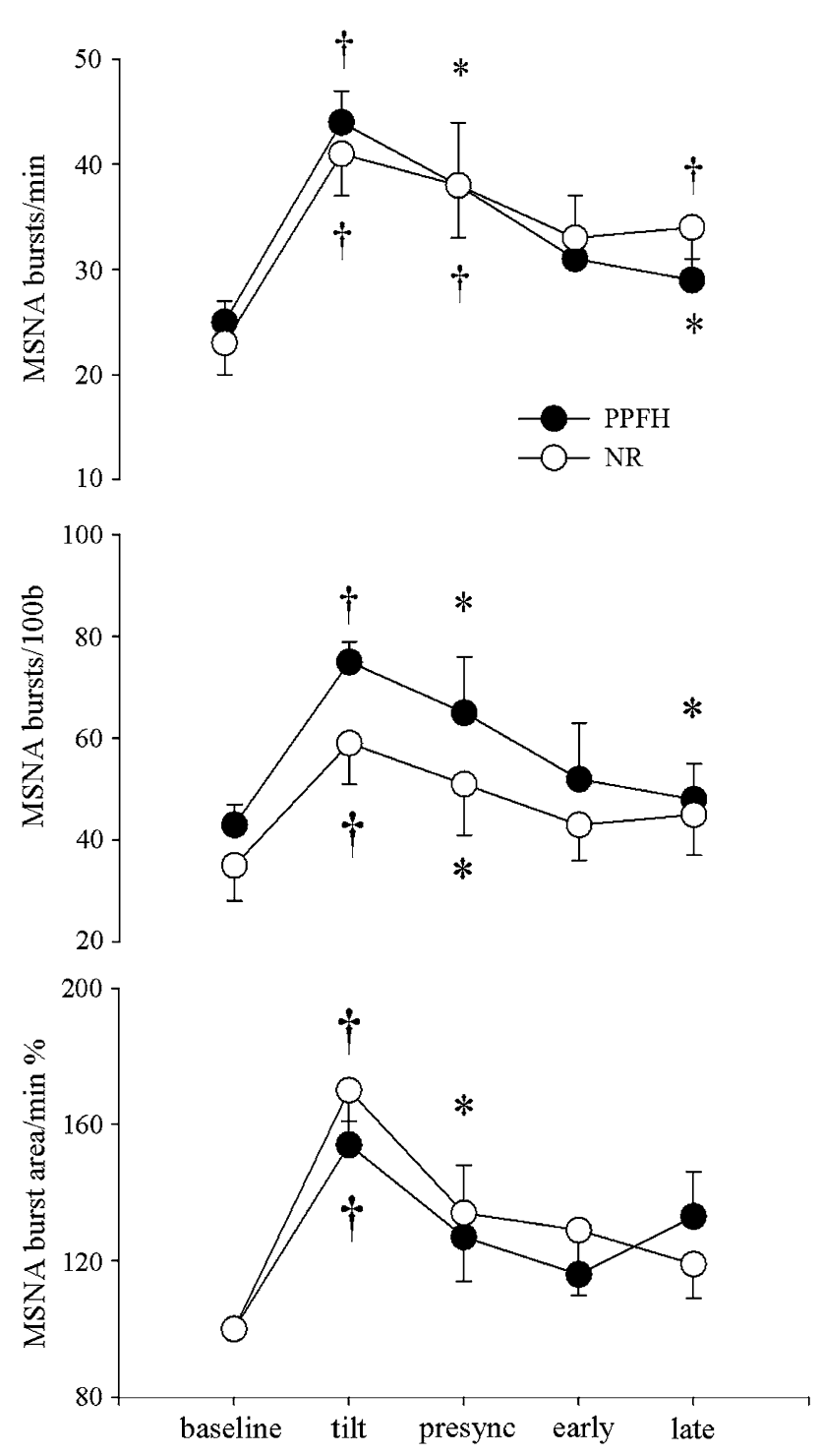

Fig. 3 Mean and standard error for MSNA burst frequency, burst incidence and burst area at baseline, early tilt, presyncope, early and late recovery in patients with prolonged post-faint hypotension (PPFH filled circles) and patients with normal recovery (NR open circles). Asterisks and dagger refer to $p<0.05$ and $p<0.01$ for within-group comparisons to baseline

$38 \pm 5$ bursts $/ \mathrm{min}(p=0.03), 65 \pm 11$ bursts $/ 100$ beats $(p=0.04)$, and $149 \pm 21 \%$ burst area $(p=0.06)$; NR $38 \pm 5$ bursts $/ \mathrm{min}(p=0.01), 51 \pm 10$ bursts $/ 100$ beats $(p=0.03)$ and $157 \pm 20 \%$ burst area $(p=0.02)$.

\section{Early recovery}

In both groups, MAP remained below baseline: $\mathrm{PPFH}$ $51 \pm 5(p=0.001)$ and NR $83 \pm 5 \mathrm{mmHg}(p=0.001)$. In the PPFH group, HR fell to baseline at $56 \mathrm{bpm}(p=0.24)$, while SV and CO remained below baseline at $69 \pm 6 \mathrm{ml}$ $(p=0.009)$ and $3.7 \pm 0.51 / \mathrm{min}(p=0.001)$. In the NR group SV began to increase but remained below baseline at $62 \mathrm{ml}(p=0.049)$. All MSNA indices returned to baseline levels in both groups. PPFH $31 \pm 6$ bursts/min $(p=0.09)$, $52 \pm 11$ bursts $/ 100$ beats $(p=0.11)$ and $133 \pm 13 \%$ burst area $(p=0.16)$; NR $33 \pm 3$ bursts $/ \min (p=0.06), 43 \pm 7$ bursts $/ 100$ beats $(p=0.25), 129 \pm 19 \%$ burst area $(p=$ $0.16)$.

\section{Late recovery}

MAP remained below baseline in both groups: $64 \pm 5$ and $93 \pm 5 \mathrm{mmHg}$, respectively ( $p=0.001, p=0.03)$. In the PPFH group, SV recovered but CO remained below baseline at $88 \pm 6 \mathrm{ml}(p=0.178)$ and $4.61 / \mathrm{min}(p=0.049)$, respectively. In the NR group, SV increased back to baseline: $65 \mathrm{ml}(p=0.127)$. MSNA indices remained at or above baseline levels in both groups. PPFH $29 \pm 4$ bursts/ $\min (p=0.02), 48 \pm 7$ bursts $/ 100$ beats $(p=0.05)$, $133 \pm 13 \%$ burst area $(p=0.08)$ and NR $34 \pm 3$ bursts/ $\min (p=0.01), 45 \pm 8$ bursts $/ 100$ beats $(p=0.09)$, $119 \pm 10 \%$ burst area $(p=0.09)$.

In summary, MAP and HR were lower at baseline and during recovery in the PPFH group. We also observed that unlike the NR group, $\mathrm{CO}$ was depressed through recovery in the PPFH group. Although TPR was lower in the PPFH group at baseline, this was not apparent during recovery and exaggerated MSNA withdrawal was not seen.

\section{Discussion}

Our hypothesis, that MSNA would remain increased from baseline values during PPFH, is supported by our findings. TPR remained constant at baseline levels in both groups, while MSNA tended to be elevated. Thus although blood pressure was clearly lower in the PPFH group during recovery, it did not appear to be mediated by an exaggerated withdrawal of MSNA. Delayed recovery of CO and a lower HR throughout recovery secondary to increased vagal outflow is therefore the most likely mechanism for PPFH. These results are consistent with the findings of Wieling et al. [19] who first described PPFH in a group of seven patients during HUT. Wieling concluded that decreased LV contractility, secondary to exaggerated vagal activity was responsible for PPFH. Like his patients, ours were predominantly middle-aged males, with low-normal resting BP, some of whom had blood phobia. Unfortunately, our methods for deriving SV, CO and TPR did not allow reliable comparisons of baseline levels so we are uncertain as to the hemodynamic mechanism for the lownormal resting BP in the PPFH group. Although MSNA is an important determinant of TPR in the dynamic setting [10], the relationship between MSNA and TPR at rest is 
more complicated, particularly with increasing age [27]. We measured similar MSNA levels in the two groups at baseline and throughout tilt, therefore, we think TPR was unlikely to be lower in the PPFH group. Therefore, our data do not support the hypothesis that PPFH patients have low resting TPR, nor impaired sympatho-vasoconstriction predisposing them to VVS [8, 28].

Even though MSNA has been studied extensively during tilt and presyncope [5-8, 28, 30], few studies include MSNA during recovery [13, 30]. This is because it is technically very difficult to maintain a satisfactory nerve recording field during the course of presyncope and recovery. The recording electrode may be dislodged by muscle tensing, myoclonic leg movements or the tilt-back maneuver. In some studies, the resulting decrease in burst frequency or size may have been falsely interpreted as sympathetic withdrawal [31]. The only way to be sure that a field has been maintained throughout presyncope is to observe spontaneous recovery of the bursting pattern after tilt-back without adjusting the recording electrode [8]. Although MSNA remained well above baseline during presyncope, we observed a terminal fall-off in MSNA immediately before tilt-back in both groups. This data confirms previous studies showing that provided patients became sufficiently hypotensive, some degree of sympathetic withdrawal usually occurred during the final seconds of presyncope before tilt-back [5, 8, 13, 28]. This is partly because individual bursts of MSNA are gated to the diastolic phase of the cardiac cycle and when heart rate decreases, burst rate follows [32]. Two recent studies have contested this finding [29, 31].We observed that the decrease in MSNA burst rate was not totally dependent on bradycardia because burst incidence usually decreased in unison with burst rate. For ethical reasons, and to decrease the risk of losing the recording field, patients were tilted back to the horizontal before bradycardia and complete sympathetic withdrawal ensued. Consequently, we demonstrated only partial sympathetic withdrawal at tilt-back and thereafter, maintenance of MSNA at or above baseline levels during recovery. We are uncertain as to why MSNA was not higher in the PPFH group at this time and cannot exclude some degree of central sympathetic depression. Nevertheless, our data demonstrated delayed recovery of $\mathrm{CO}$ and lower HR in the PPFH group, which implies that this is the most likely mechanism responsible. Therefore, doctors with a specialist interest in syncope should be aware that a minority of fainters $(7.4 \%$ in this study and $1.9 \%$ in the study by Wieling et al. [19] may exhibit PPFH, for example during tilt-testing and so will require monitoring and rest in the horizontal position for an extended recovery period. This phenomenon seems to be secondary to a transient vagal-induced paresis of the left ventricle [19], which is a fascinating and novel physiological prospect. We predict that passive leg raising, a manoeuvre that rapidly increases venous return, will not decrease recovery time during $\mathrm{PPFH}$. A central stimulation by "dynamic tension" (active bilateral leg flexion and extension) may be required [19] (Wieling et al. 2012 Case).

\section{Limitations}

This is a retrospective study, looking at a relatively small group of patients, which was limited mainly by technical difficulties obtaining satisfactory MSNA data. At the time the recordings were made, we were mainly concerned with preserving the recording field throughout presyncope, and so we did not allow our patients to become as bradycardic as the PPFH group previously reported [19]. This accounts for the difference in hemodynamics between the two studies. Nevertheless, this should not detract from our main finding, that MSNA is not decreased during PPFH. MSNA recordings, although the best dynamic measure of sympatho-vasoconstriction, are all limited to the vascular bed of one (lower) limb, which may not be representative of the major resistance/capacitance vessels during vasovagal syncope [33]. We did not measure lower limb blood flow and so cannot exclude loss of sympathetic transduction or active vasodilatation as a possible mechanism for increased MSNA during PPFH [31].

Open Access This article is distributed under the terms of the Creative Commons Attribution License which permits any use, distribution, and reproduction in any medium, provided the original author(s) and the source are credited.

\section{References}

1. van Lieshout JJ, Wieling W, Karemaker JM, Eckberg D (1991) The vasovagal response. Clin Sci 81:575-586

2. Barcroft H, Edhholm OG (1945) On the vasodilatation in human skeletal muscle during post-hemorrhagic fainting. J Physiol 104:161-175

3. Brigden W, Howarth S, Sharpey-Schafer EP (1950) Postural changes in the peripheral blood-flow of normal subjects with observations on vasovagal fainting reactions as a result of tilting, the lordotic posture, pregnancy and spinal anaesthesia. Clin Sci 9:71-91

4. Dietz NM, Halliwill JR, Spielmann JM, Lawler LA, Papouchado B, Eickhoff TJ, Joyner MJ (1997) Sympathetic withdrawal and forearm vasodilatation during vasovagal syncope in humans. J Appl Physiol 82:1785-1793

5. Jardine DL, Ikram H, Frampton CM, Frethey R, Bennett SI, Crozier IG (1998) Autonomic control of vasovagal syncope. Am J Physiol Heart Circ Physiol 274:H2110-H2115

6. Kamiya A, Hayano J, Kawada T, Michikami D, Yamamoto K, Ariumi H, Shimizu S, Uemura K, Miyamoto T, Aiba T, Sunagawa K, Sugimachi M (2005) Low-frequency oscillation of sympathetic nerve activity decreases during development of tilt-induced syncope preceding sympathetic withdrawal and bradycardia. Am J Physiol Heart Circ Physiol 289:H1758-H1769 
7. Morillo CA, Eckberg DL, Ellenbogen KA, Beightol LA, Hoag JB, Tahvanainen KUO, Kuusela TA, Diedrich AM (1997) Vagal and sympathetic mechanisms in patients with orthostatic vasovagal syncope. Circulation 96:2509-2513

8. Mosqueda-Garcia R, Furlan R, Fernandez-Violante R, Desai T, Snell M, Jarai Z, Ananthram V, Robertson RM, Robertson D (1997) Sympathetic and baroreceptor reflex function in neurally mediated syncope evoked by tilt. J Clin Invest 99:2736-2744

9. Wallin BG, Sundlof G (1982) Sympathetic outflow to muscles during vasovagal syncope. J Auton Nerv Syst 6:287-291

10. Fu Q, Shook RP, Okazaki K, Hastings JL, Shibata S, Conner CL, Palmer MD, Levine BD (2006) Vasomotor sympathetic neural control is maintained during sustained upright posture in humans. J Physiol 577:679-687

11. Vissing SF, Scherrer U, Victor R (1989) Relationship between sympathetic outflow and vascular resistance in the calf during perturbations in central venous pressure. Circ Res 65:1710-1717

12. Glick G, Yu PN (1963) Hemodynamic changes during spontaneous vasovagal reactions. Am J Med 34:42-51

13. Jardine DL, Melton IC, Crozier IG, English S, Bennett SI, Frampton CM, Ikram H (2001) Decrease in cardiac output and muscle sympathetic nerve activity during vasovagal syncope. Am J Physiol Heart Circ Physiol 282:H1804-H1809

14. Murray RH, Thompson LJ, Bowers JA, Albright CD (1968) Heamodynamic effects of graded hypovolemia and vasodepressor syncope induced by lower body negative pressure. Am Heart $\mathbf{J}$ 76:799-811

15. Stevens PM (1966) Cardiovascular dynamics during orthostasis and influence of intravascular instrumentation. Am J Cardiol $17: 211-218$

16. Verheyden B, Liu J, van Dijk N, Westerhof BE, Reybrouck T, Aubert AE, Wieling W (2008) Steep fall in cardiac output is main determinant of hypotension during drug-free and nitroglycerineinduced orthostatic vasovagal syncope. Heart Rhythm 5:16951701

17. Weissler AM, Warren JV, Estes EH, McIntosh HD, Leonard JJ (1957) Vasodepressor syncope. Circulation 15:875-882

18. Krediet CTP, van Dijk N, Linzer M, van Lieshout JJ, Wieling W (2002) Management of vasovagal syncope: controlling or aborting faints by leg crossing and muscle tensing. Circulation 106:1684-1689

19. Wieling W, Rozenberg J, Schon IK, Karemaker JM, Westerhof BE, Jardine DL (2011) Prolonged post-faint hypotension can be reversed by dynamic tension. Clin Auton Res 21(6):415-418. doi: 10.1007/s10286-011-0133-7

20. Bartoletti A, Alboni P, Ammirati F et al (2000) "The Italian Protocol": a simplified head-up tilt testing potentiated with oral nitroglycerin to assess patients with unexplained syncope. Europace 2:339-342

21. Hagbarth KE, Valbo AB (1967) Mechanoreceptor activity recorded percutaneously with semi-electrodes in human peripheral nerves. Physiol Scand 69:121-122

22. Wesseling KH (1995) A century of non-invasive arterial pressure measurement: from Marey to Penaz and finapres. Homeostasis 36:50-66

23. Jellema WT, Imholz BPM, van Goudoever J, Wesseling KH, van Lieshout JJ (1996) Finger arterial versus intrabrachial pressure and continuous cardiac output during head-up tilt testing in healthy subjects. Clin Sci 91:193-200

24. Langewouters GJ, Wesseling KH, Goedhard WJA (1984) The elastic properties of 45 human thoracic and 20 abdominal aortas in vitro and the parameters of a new model. J Biotech 17:425-535

25. Harms MPM, Wesseling KH, Pott F, Jenstrup M, van Goudoever J, Secher NH, van Lieshout JJ (1999) Continuous stroke volume monitoring by modelling flow from non-invasive measurement of arterial pressure in humans under orthostatic stress. Clin Sci 97:291-301

26. Stok WJ, Baisch F, Hillebrecht A (1993) Non-invasive cardiac output measurement by arterial pulse analysis compared with inert gas rebreathing. J Appl Physiol 74:2687-2693

27. Charkhoudian N, Joyner MJ, Johnson CP, Eisenach JH, Dietz NM, Wallin BG (2005) Balance between cardiac output and sympathetic nerve activity in resting humans: role in arterial pressure regulation. J Physiol 568:315-321

28. Vaddadi G, Lambert E, Corcoran SJ, Esler MD (2007) Postural syncope: mechanisms and management. Med J Aust 187:299-304

29. Cooke WH, Rickards CA, Ryan KL, Kuusela TA, Convertino VA (2009) Muscle sympathetic activity during intense lower body negative pressure to presyncope in humans. J Physiol 587:49874999

30. Iwase S, Mano T, Kamiya A, Niimi Y, Suzumara A (2002) Syncopal attack alters the burst properties of muscle sympathetic nerve activity in humans. Autonom Neurosci 95:141-145

31. Vaddadi G, Esler MD, Dawood T, Lambert E (2010) Persistence of muscle sympathetic nerve activity during vasovagal syncope. Eur Heart J 16:2027-2033

32. Malpas SC (1998) The rhythmicity of sympathetic nerve activity. Prog Neurobiol 56:65-96

33. Hirsch AT, Levenson DJ, Cutler SS, Dzau VJ, Creager MA (1989) Regional vascular responses to prolonged lower body negative pressure in normal subjects. Am J Physiol 257:H219$\mathrm{H} 225$ 